\title{
Higgs sector of the minimal left-right symmetric model
}

\author{
Gabriela Barenboim, ${ }^{1}$ Martin Gorbahn, ${ }^{2}$ Ulrich Nierste, ${ }^{3}$ and Martti Raidal ${ }^{3,4}$ \\ ${ }^{1}$ Fermi National Accelerator Laboratory, Batavia, Illinois 60510-500 \\ ${ }^{2}$ Physik-Department, Technische Universität München, D-85748 Garching, Germany \\ ${ }^{3}$ CERN-Theory, CH-1211 Geneva 23, Switzerland \\ ${ }^{4}$ National Institute of Chemical Physics and Biophysics, Tallinn 10143, Estonia
}

(Received 12 July 2001; published 18 April 2002)

\begin{abstract}
We perform an exhaustive analysis of the most general Higgs sector of the minimal left-right symmetric model (MLRM). We find that the $C P$ properties of the vacuum state are connected to the Higgs spectrum: if $C P$ is broken spontaneously, the MLRM does not approach the standard model in the limit of a decoupling left-right symmetry breaking scale. Depending on the size of the $C P$ phases, scenarios with extra nondecoupling flavor-violating doublet Higgs bosons or very light SU(2) triplet Higgs bosons emerge, both of which are ruled out by phenomenology. For zero $C P$ phases the nonstandard Higgs bosons decouple only if a very unnatural fine-tuning condition is satisfied. We also discuss generalizations to the nonminimal Higgs sector.
\end{abstract}

DOI: 10.1103/PhysRevD.65.095003

PACS number(s): 12.60.Cn, 11.30.Er, 14.80.Cp

\section{INTRODUCTION}

Left-right symmetric models are extensions of the standard model (SM) based on the gauge group $\mathrm{SU}(2)_{R}$ $\times \mathrm{SU}(2)_{L} \times \mathrm{U}(1)_{B-L}[1]$. The right-handed fermion fields are $\mathrm{SU}(2)_{R}$ doublets and parity $P$ is an exact symmetry of the Lagrangian. At a high scale $v_{R}$ well above the electroweak breaking scale, $\mathrm{SU}(2)_{R} \times \mathrm{SU}(2)_{L} \times \mathrm{U}(1)_{B-L} \times P$ is spontaneously broken to the $\mathrm{SM}$ gauge group $\mathrm{SU}(2)_{L}$ $\times \mathrm{U}(1)_{Y}$. The $\mathrm{U}(1)$ charges have a physical interpretation as the difference $B-L$ of baryon and lepton number. The hypercharge $Y$, which is an ad hoc quantum number of the SM, emerges as the combination $Y=\mathrm{T}_{3, R}+(B-L) / 2$, where $\mathrm{T}_{3, R}$ is the third component of the right-handed isospin. The Higgs sector of the minimal left-right symmetric model (MLRM) consists of two Higgs triplets $\Delta_{R}, \Delta_{L}$ and a bidoublet $\Phi$. The neutral component of $\Delta_{R}$ acquires a vacuum expectation value $(\mathrm{VEV}) v_{R}$, which breaks the $\mathrm{SU}(2)_{R}$ and $P$ symmetries. The bidoublet $\Phi$ breaks the electroweak symmetry down to $U(1)_{e m}$. The choice of Higgs triplets $\Delta_{L, R}$ rather than doublets permits a Majorana mass term and thereby small neutrino masses via the seesaw mechanism [2]. This requires $v_{R}$ to be very high, typically of order $10^{10}$ $-10^{15} \mathrm{GeV}$.

\section{SPONTANEOUS CP VIOLATION}

Models with explicit $C P$ violation suffer from a general problem: a $C P$-noninvariant Lagrangian usually contains too many sources of $C P$ violation. In most extensions of the SM, especially in the MSSM, this problem becomes very severe: some $C P$-violating phases must be fine-tuned to comply with the observed smallness of $C P$ violating observables. Models with spontaneous $C P$ violation $(\mathrm{SCPV})$ are therefore an attractive alternative, because their only few sources of $C P$ violation are the complex phases of the VEVs of Higgs fields. The MLRM with SCPV has recently attracted new attention in the context of $C P$ phenomenology probed in current experiments $[3,4]$. The MLRM with SCPV has the attractive feature that $C, P$, and $T$ are all exact symmetries of the Lagrangian. Both the MLRM with and without SCPV have been studied extensively. Yet its complicated Higgs sector has never been analyzed completely. In [3-5] the case of sizable $C P$-violating phases was considered aiming at the explanation of the observed Cabibbo-Kobayashi-Maskawa (CKM) $C P$ phase through SCPV. In [6,7], however, large $C P$-violating phases of the VEVs have been discarded because of fine-tuning arguments. We will clarify this point in the following.

\section{MODEL}

The $\mathrm{SU}(2)_{L} \times \mathrm{SU}(2)_{R} \times \mathrm{U}(1)_{B-L}$ charge assignments for the quark and lepton multiplets are $Q_{L}(1 / 2,0,1 / 3)$, $Q_{R}(0,1 / 2,1 / 3), L_{L}(1 / 2,0,-1)$ and $L_{R}(0,1 / 2,-1)$. The Higgs multiplets are

$$
\Phi=\left(\begin{array}{cc}
\phi_{1}^{0} & \phi_{1}^{+} \\
\phi_{2}^{-} & \phi_{2}^{0}
\end{array}\right), \quad \Delta_{L, R}=\left(\begin{array}{cc}
\delta_{L, R}^{+} / \sqrt{2} & \delta_{L, R}^{++} \\
\delta_{L, R}^{0} & -\delta_{L, R}^{+} / \sqrt{2}
\end{array}\right)
$$

They transform under $\mathrm{U}_{L, R} \in \mathrm{SU}(2)_{\mathrm{L}, \mathrm{R}}$ as $\Phi \rightarrow U_{L} \Phi U_{R}^{\dagger}$, $\Delta_{L} \rightarrow U_{L} \Delta_{L} U_{L}^{\dagger}$ and $\Delta_{R} \rightarrow U_{R} \Delta_{R} U_{R}^{\dagger}$. The Higgs fields transform under parity as $\Delta_{L} \leftrightarrow \Delta_{R}$ and $\phi \leftrightarrow \phi^{\dagger}$. Their charge conjugation transformation reads

$$
\mathrm{C}_{\phi}: \quad \phi \leftrightarrow \widetilde{\phi}=\tau_{2} \phi^{*} \tau_{2}, \quad \mathrm{C}_{\Delta}: \Delta \leftrightarrow \widetilde{\Delta}=\tau_{2} \Delta^{*} \tau_{2} .
$$

The most general $C \times P$-invariant Higgs potential is [6] 


$$
\begin{aligned}
V\left(\Delta_{R}, \Delta_{L}, \Phi\right)= & -\mu_{1}^{2} \operatorname{Tr}\left(\phi^{\dagger} \phi\right)-\mu_{2}^{2}\left[\operatorname{Tr}\left(\widetilde{\phi} \phi^{\dagger}\right)+\operatorname{Tr}\left(\widetilde{\phi}^{\dagger} \phi\right)\right]-\mu_{3}^{2}\left[\operatorname{Tr}\left(\Delta_{L} \Delta_{L}^{\dagger}\right)+\operatorname{Tr}\left(\Delta_{R} \Delta_{R}^{\dagger}\right)\right]+\lambda_{1}\left[\operatorname{Tr}\left(\phi \phi^{\dagger}\right)\right]^{2} \\
& +\lambda_{2}\left\{\left[\operatorname{Tr}\left(\widetilde{\phi} \phi^{\dagger}\right)\right]^{2}+\left[\operatorname{Tr}\left(\widetilde{\phi}^{\dagger} \phi\right)\right]^{2}\right\}+\lambda_{3}\left[\operatorname{Tr}\left(\widetilde{\phi} \phi^{\dagger}\right) \operatorname{Tr}\left(\widetilde{\phi}^{\dagger} \phi\right)\right]+\lambda_{4}\left\{\operatorname{Tr}\left(\phi \phi^{\dagger}\right)\left[\operatorname{Tr}\left(\widetilde{\phi} \phi^{\dagger}\right)+\operatorname{Tr}\left(\widetilde{\phi}^{\dagger} \phi\right)\right]\right\} \\
& +\rho_{1}\left\{\left[\operatorname{Tr}\left(\Delta_{L} \Delta_{L}^{\dagger}\right)\right]^{2}+\left[\operatorname{Tr}\left(\Delta_{R} \Delta_{R}^{\dagger}\right)\right]^{2}\right\}+\rho_{2}\left[\operatorname{Tr}\left(\Delta_{L} \Delta_{L}\right) \operatorname{Tr}\left(\Delta_{L}^{\dagger} \Delta_{L}^{\dagger}\right)+\operatorname{Tr}\left(\Delta_{R} \Delta_{R}\right) \operatorname{Tr}\left(\Delta_{R}^{\dagger} \Delta_{R}^{\dagger}\right)\right] \\
& +\rho_{3}\left[\operatorname{Tr}\left(\Delta_{L} \Delta_{L}^{\dagger}\right) \operatorname{Tr}\left(\Delta_{R} \Delta_{R}^{\dagger}\right)\right]+\rho_{4}\left[\operatorname{Tr}\left(\Delta_{L} \Delta_{L}\right) \operatorname{Tr}\left(\Delta_{R}^{\dagger} \Delta_{R}^{\dagger}\right)+\operatorname{Tr}\left(\Delta_{L}^{\dagger} \Delta_{L}^{\dagger}\right) \operatorname{Tr}\left(\Delta_{R} \Delta_{R}\right)\right]+\alpha_{1}\left\{\operatorname{Tr}\left(\phi \phi^{\dagger}\right)\right. \\
& \left.\times\left[\operatorname{Tr}\left(\Delta_{L} \Delta_{L}^{\dagger}\right)+\operatorname{Tr}\left(\Delta_{R} \Delta_{R}^{\dagger}\right)\right]\right\}+\alpha_{2}\left[\operatorname{Tr}\left(\phi \widetilde{\phi}^{\dagger}\right)+\operatorname{Tr}\left(\phi^{\dagger} \widetilde{\phi}\right)\right]\left[\operatorname{Tr}\left(\Delta_{R} \Delta_{R}^{\dagger}\right)+\operatorname{Tr}\left(\Delta_{L} \Delta_{L}^{\dagger}\right)\right]+\alpha_{3}\left[\operatorname{Tr}\left(\phi \phi^{\dagger} \Delta_{L} \Delta_{L}^{\dagger}\right)\right. \\
& \left.+\operatorname{Tr}\left(\phi^{\dagger} \phi \Delta_{R} \Delta_{R}^{\dagger}\right)\right]+\beta_{1}\left[\operatorname{Tr}\left(\phi \Delta_{R} \phi^{\dagger} \Delta_{L}^{\dagger}\right)+\operatorname{Tr}\left(\phi^{\dagger} \Delta_{L} \phi \Delta_{R}^{\dagger}\right)\right]+\beta_{2}\left[\operatorname{Tr}\left(\widetilde{\phi} \Delta_{R} \phi^{\dagger} \Delta_{L}^{\dagger}\right)+\operatorname{Tr}\left(\widetilde{\phi}^{\dagger} \Delta_{L} \phi \Delta_{R}^{\dagger}\right)\right] \\
& +\beta_{3}\left[\operatorname{Tr}\left(\phi \Delta_{R} \widetilde{\phi}^{\dagger} \Delta_{L}^{\dagger}\right)+\operatorname{Tr}\left(\phi^{\dagger} \Delta_{L} \widetilde{\phi} \Delta_{R}^{\dagger}\right)\right] .
\end{aligned}
$$

Here all coefficients are real. We discuss the case of a $C P$ non-invariant Higgs potential later. The coefficients in Eq. (2) must be such that $V$ has a nontrivial minimum which leaves $U(1)_{e m}$ unbroken. By using the broken symmetries one can arrange the VEVs such as $[1,6]$

$$
\begin{aligned}
& \left\langle\phi_{1}^{0}\right\rangle=\frac{k_{1}}{\sqrt{2}},\left\langle\phi_{2}^{0}\right\rangle=\frac{k_{2}}{\sqrt{2}} e^{i \alpha}, \\
& \left\langle\delta_{L}^{0}\right\rangle=\frac{v_{L}}{\sqrt{2}} e^{-i \theta},\left\langle\delta_{R}^{0}\right\rangle=\frac{v_{R}}{\sqrt{2}},
\end{aligned}
$$

with real and positive $v_{L, R}$ and $k_{1,2}$. If $\beta_{i}, \rho_{i}=\mathcal{O}(1)$, the condition $v_{R} \gg k_{1,2}$ automatically enforces $k_{1} k_{2} /\left(v_{L} v_{R}\right)$ to be of order 1. This VEV seesaw mechanism [6] suppresses $v_{L}$, as needed to comply with the data on the electroweak $\rho$ parameter. The Yukawa Lagrangian reads

$$
-L_{Y}=\bar{Q}_{L} \hat{F} \phi Q_{R}+\bar{Q}_{L} \hat{G} \widetilde{\phi} Q_{R}+\text { H.c. }
$$

Here $\hat{F}$ and $\hat{G}$ are $3 \times 3$ matrices in flavor space. If $L_{Y}$ conserves $C P$, one can choose them real and symmetric. Since $\phi_{1}^{0}$ and $\phi_{2}^{0}$ couple to both up and down quarks there will be flavor-changing neutral couplings. Here it is useful to define $[5]$

$$
\left(\begin{array}{c}
\phi_{-}^{0} \\
\phi_{+}^{0}
\end{array}\right)=\left(\begin{array}{cc}
\cos \beta & \sin \beta e^{i \alpha} \\
-\sin \beta e^{-i \alpha} & \cos \beta
\end{array}\right)\left(\begin{array}{c}
\phi_{1}^{0} \\
\phi_{2}^{0 *}
\end{array}\right),
$$

with $\tan \beta=k_{2} / k_{1}$. Analogously we define $\left(\phi_{-}^{+}, \phi_{+}^{+}\right)$. The imaginary part of the flavor-conserving field $\phi_{-}^{0}$ becomes a component of the Goldstone bosons absorbed by Z's. The orthogonal combination $\phi_{+}^{0}$ has flavor-changing neutral couplings. For example the flavor-changing couplings to the down quarks are given by

$$
\mathcal{L}^{F C \phi^{0} d}=\sqrt{2} \frac{k_{+}}{k_{-}^{2}} \phi_{+}^{0 *} \bar{D}_{L} V_{L}^{\dagger} M_{u} V_{R} D_{R},
$$

where $k_{ \pm}^{2}=k_{1}^{2} \pm k_{2}^{2}$ with the electroweak breaking scale $k_{+}$ $\simeq 246 \mathrm{GeV} . P$ and $C P$ invariance implies $\left|V_{L}\right|=\left|V_{R}\right|$ and calculable phases for the left and right-handed CKM matrices. If $P$ and $C P$ are broken spontaneously, the masses of the flavor-violating Higgs bosons must exceed $10 \mathrm{TeV}$ from phenomenology [4]. If one relaxes the $C P$ invariance of $L_{Y}$, the phases of $V_{R}$ become independent of $V_{L}$ and generically this lower bound becomes much stronger. Hence the mass of the flavor-changing Higgs boson must be determined by $v_{R}$ rather than $k_{+}$. The $C P$-violating complex phase $\alpha$ enters the quark mass matrix through the Yukawa interactions in Eq. (3). If $L_{Y}$ in Eq. (3) is chosen to conserve $C P$, the CKM $C P$ violation stems solely from $\alpha \neq 0$. This case requires that $k_{1} k_{2}(\sin \alpha) / k_{-}^{2} \approx m_{b} / m_{t}$ [3-5]. In [3-5] this has been achieved by choosing $k_{2} / k_{1} \leqslant \mathcal{O}\left(m_{b} / m_{t}\right)$ and $\alpha=\mathcal{O}(1)$.

We next decompose the Higgs fields into real and imaginary parts: $\phi_{1}^{0}=\left(\phi_{1}^{0 r}+i \phi_{1}^{0 i}+k_{1}\right) / \sqrt{2}, \quad \phi_{2}^{0}=\left(\phi_{2}^{0 r}+i \phi_{2}^{0 i}\right.$ $\left.+k_{2}\right) \exp (i \alpha) / \sqrt{2}$ and analogously $\Delta_{L}^{0}$ and $\Delta_{R}^{0}$. $V$ in Eq. (2) is minimized by solving the equations

$$
\frac{\partial V}{\partial \phi_{1}^{0 r}}=\frac{\partial V}{\partial \phi_{2}^{0 r}}=\frac{\partial V}{\partial \phi_{2}^{0 i}}=\frac{\partial V}{\partial \Delta_{R}^{0 r}}=\frac{\partial V}{\partial \Delta_{L}^{0 r}}=\frac{\partial V}{\partial \Delta_{L}^{0 i}}=0 .
$$

In general Eq. (6) expresses six chosen parameters of $V$ in terms of the remaining parameters and $k_{1}, k_{2}, \alpha, v_{R}, v_{L}$ and $\theta$. Yet there is an important exception: if the parameters in $V$ are such that $C P$ remains unbroken, the complex phases $\alpha$ and $\theta$ are zero and $V$ is quadratic in $\phi_{1,2}^{0 i}$. The rank of Eq. (6) then collapses to 4 allowing us to solve for only 4 parameters in terms of $k_{1}, k_{2}, v_{R}$ and $v_{L}$. For generic choices of the parameters in $V$ the polynomial equations in Eq. (6) will not generate the desired gauge hierarchy $v_{R} \gg k_{+}$. This relation must be encoded in $V$ : either some ratio of dimensionful parameters $\mu_{i}^{2}$ or some coupling or a combination of couplings must be chosen small to define the ratio $k_{+}^{2} / v_{R}^{2}$. From this consideration it is clear that fine-tuning is unavoidable. Parameters fine-tuned to small values must be protected by an approximate symmetry, otherwise the corresponding solution becomes unstable under radiative corrections.

Our strategy is to expand the solutions of Eq. (6) and the Higgs boson mass matrices in terms of $\epsilon=\max \left\{k_{+} /\right.$ $\left.v_{R}, v_{L} / k_{+}\right\}$. Thereby we determine the Higgs spectrum in the decoupling limit. We will see that there are different possibilities to generate the gauge hierarchy: depending on which 
parameter is chosen to be of order $\epsilon^{2}$, different low energy models emerge in the decoupling limit. We first use the derivatives with respect to $\phi_{1,2}^{0 r}$ and $\Delta_{R}^{0 r}$ in Eq. (6) to find

$$
\frac{\mu_{1}^{2}}{v_{R}^{2}} \simeq \frac{\alpha_{1}}{2}-\frac{\alpha_{3} k_{2}^{2}}{2 k_{-}^{2}}, \quad \frac{\mu_{2}^{2}}{v_{R}^{2}} \simeq \frac{\alpha_{2}}{2}+\frac{\alpha_{3} k_{1} k_{2}}{4 k_{-}^{2} \cos \alpha}, \quad \frac{\mu_{3}^{2}}{v_{R}^{2}} \simeq \rho_{1} .
$$

These relations are valid in all scenarios discussed in the following. Here and in the following " $\simeq$ " means "equal up to corrections of order $\epsilon^{2}$." Next we calculate the mass matrices from the second derivatives of $V$ with respect to the Higgs fields and insert the results for the six parameters found from Eq. (7). This step gives us a $2 \times 2$ mass matrix for the doubly charged Higgs fields, a $4 \times 4$ matrix for the singly charged Higgs fields and an $8 \times 8$ matrix for the neutral ones. The latter two contain 2 zero eigenvalues each corresponding to the pseudo-Goldstone modes absorbed by the left- and right-handed vector bosons. A pivotal role for the mass spectrum is played by the term involving $\alpha_{3}$ in $V$. It is the only term which generates a mass splitting of order $v_{R}^{2}$ between the bidoublet fields:

$$
\alpha_{3} \operatorname{Tr}\left(\phi^{\dagger} \phi \Delta_{R} \Delta_{R}^{\dagger}\right) \rightarrow \alpha_{3}\left|v_{R}\right|^{2}\left(\left|\phi_{2}^{+}\right|^{2}+\left|\phi_{2}^{0}\right|^{2}\right) / 2 .
$$

Here the components of $\phi_{ \pm}=\left(\phi_{ \pm}^{0}, \phi_{ \pm}^{+}\right)$are defined in Eq. (4). Up to corrections of order $\epsilon^{2}$ the fields of $\phi_{-}$become components of the Goldstones absorbed by $W_{L}$ and $Z_{L}$ and of light Higgs particles, whose masses are of order $k_{+}$or smaller. Hence if $\alpha_{3}=\mathcal{O}(1)$, the bidoublet components in $\phi_{+}$, acquire masses of order $v_{R}$. However, we will be frequently led to scenarios with $\alpha_{3}=\mathcal{O}\left(\epsilon^{2}\right)$. Then all entries for the bidoublet mass matrices are at most of order $k_{+}^{2}$. From Eq. (2) one easily verifies that the terms which mix bidoublet and triplet fields are $\mathcal{O}\left(v_{R} k_{+}\right)$or smaller. This form of the mass matrices implies that the neutral (charged) Higgs sector has at least four (two) physical Higgs boson masses of order $k_{+}$. Up to terms of order $\epsilon$ the corresponding mass eigenstates are bidoublet fields. That is, for $\alpha_{3}=\mathcal{O}\left(\epsilon^{2}\right)$ one encounters a two-Higgs doublet model (2HDM) in the decoupling limit $v_{R} \rightarrow \infty$. In view of the flavor-changing couplings in Eq. (5) such scenarios are unacceptable.

\section{SCENARIOS WITH $v_{L}=0$}

We will study the $v_{L}=0$ scenario here for two reasons: first, it has been used extensively in the literature $[6,9,10]$, and second, most of the characteristic features of the general case can be studied from this simplified case. In addition to Eqs. (7) the minimization conditions in Eq. (6) give

$$
\alpha_{3}=4 \tilde{\lambda} \frac{k_{-}^{2}}{v_{R}^{2}}, \quad \beta_{1}=-2 \beta_{3} \frac{k_{2}}{k_{1}} \cos \alpha, \quad \beta_{2}=\beta_{3} \frac{k_{2}^{2}}{k_{1}^{2}},
$$

where $\tilde{\lambda}=2 \lambda_{2}-\lambda_{3}$. It must be clear that the scenario with $v_{L}=0$ is singular, because the six equations in Eq. (6) involve only 4 parameters $k_{1}, k_{2}, \alpha$ and $v_{R}$. After eliminating $k_{1}, k_{2}, \alpha$ and $v_{R}$ from Eqs. (9) one finds two relations be- tween the coefficients of $V$. In $[6,9,10]$ this problem has been circumvented by choosing $\beta_{1,2,3}=0$. Since there is no suitable symmetry, this scenario cannot be stabilized after renormalization. In particular the desired Majorana couplings induce non-zero $\beta_{i}$-terms at the loop level. After eliminating the $\mu_{i}$ 's with Eqs. (7) we find the physical eigenvalues of the singly charged mass matrix as

$$
M_{1}^{+2} \simeq \frac{\alpha_{3}}{2} v_{R}^{2} \frac{k_{+}^{2}}{k_{-}^{2}}, \quad M_{2}^{+2} \simeq \frac{v_{R}^{2}}{2}\left(\rho_{3}-2 \rho_{1}\right) .
$$

Next we use Eqs. (9) to eliminate $\alpha_{3}$. Since $\alpha_{3}$ is of order $\epsilon^{2}$, this immediately implies that $M_{1}^{+}$in Eqs. (10) is of order $k_{+}$, not of order $v_{R}$. (The smallness of $\alpha_{3}$ can be motivated by an approximate discrete symmetry: $V$ is invariant under $C \equiv C_{\phi^{\circ}} C_{\Delta}$ [defined in Eq. (1)]. Demanding invariance of $V$ under $C_{\phi} \times C_{\Delta}$ implies $\alpha_{3}=0$ (and $\beta_{2}=\beta_{3}$ ).) The smallness of $M_{1}^{+}$simply reflects our finding that $\alpha_{3}=\mathcal{O}\left(\epsilon^{2}\right)$ leads to a $2 \mathrm{HDM}$ in the decoupling limit, as discussed after (8). The peculiar result for $\alpha_{3}$ stems from $\partial V / \partial \phi_{2}^{0 i}$ in Eqs. (7). The mechanism here is the following: first the heavy scale $v_{R}$ is defined by the size of the dimensionful parameters $\mu_{i}$ in $V$. Then the first equation in Eqs. (9) tells us that in the chosen scenario (with $v_{L}=0$ and $\alpha \neq 0$ ) the electroweak scale is defined by $k_{+}^{2}=\mathcal{O}\left(\alpha_{3} v_{R}^{2}\right)$. Choosing $\alpha_{3}=\mathcal{O}(1)$ would fail to produce the desired gauge hierarchy $k_{+} \ll v_{R}$. The important lesson is that the same small parameter $\alpha_{3}$, which defines the ratio $k_{+} / v_{R}=\mathcal{O}(\epsilon)$ through Eqs. (9), enters the physical Higgs boson masses in Eqs. (10). Only after eliminating $\alpha_{3}$ via the minimization conditions (9) does the smallness of $M_{1}^{+}=\mathcal{O}\left(k_{+}\right)$become transparent. From the discussion preceding (8) we conclude that in the limit $v_{R} \rightarrow \infty$ the bidoublet does not decouple. What phenomenologically matters, is of course the masses of the flavor changing neutral current (FCNC) Higgs boson. We have calculated the $8 \times 8$ neutral mass matrix and have indeed verified that there are FCNC Higgs masses of order $\alpha_{3} v_{R}^{2}$ (or explicitly of order $k_{+}^{2}$ ). Even if $\tilde{\lambda}$ in Eqs. (9) is stretched to the largest values compatible with perturbation theory, the FCNC Higgs boson masses are way too small to comply with the precision data from flavor physics. In addition to the unacceptable light FCNC Higgs bosons a fine-tuning problem emerges in Eqs. (7): the terms involving $\alpha_{3}$ are now sub-leading in $\epsilon$, the dependence on $\alpha$ is lost and the three parameters $\mu_{1,2,3}$ only depend on $v_{R}$, up to $\mathcal{O}\left(\epsilon^{2}\right)$ corrections. The last two equations in Eqs. (7) now require that $\mu_{3}^{2} / \mu_{i}^{2} \simeq 2 \rho_{1} / \alpha_{i}$ for $i=1,2$.

A qualitatively new scenario, which has been studied extensively in $[6,9,10]$, is obtained, if one chooses $\alpha=0$. Now there is no SCPV and the rank of Eq. (6) collapses to four, because all equations are real. In particular the first equation in Eqs. (9) is now absent and no restrictions on $\alpha_{3}$ occur. $\alpha_{3}=\mathcal{O}(1)$ is now possible, and for this choice we can arrange for a SM-like Higgs spectrum in the decoupling limit.

We demonstrate the impact of $\alpha$ on the mass spectrum for the case $\beta_{i}=0$, which can be nicely seen from the mass term of $\phi_{2}^{0 i}=\operatorname{Im} \phi_{2}^{0}$. After using Eqs. (7) one finds 


$$
\begin{gathered}
\left.\phi_{2}^{0 i} \frac{\partial V}{\partial \phi_{2}^{0 i}}\right|_{\phi_{2}^{0 i}=0}+\left.\frac{\phi_{2}^{0 i 2}}{2} \frac{\partial^{2} V}{\partial \phi_{2}^{0 i 2}}\right|_{\phi_{2}^{0 i}=0} \\
=\phi_{2}^{0 i} k_{2} \sin \alpha\left[\frac{\alpha_{3}}{2} v_{R}^{2}-2\left(2 \lambda_{2}-\lambda_{3}\right) k_{-}^{2}\right] \\
+\phi_{2}^{0 i 2}\left[\frac{\alpha_{3}}{4} v_{R}^{2}+k_{2}^{2} \sin ^{2} \alpha-k_{-}^{2}\left(2 \lambda_{2}-\lambda_{3}\right)\right] .
\end{gathered}
$$

For $\alpha \neq 0$ the fourth minimization equation (with respect to $\left.\phi_{2}^{0 i}\right)$ enforces the linear term to vanish yielding the condition for $\alpha_{3}$ in Eqs. (9). Then the low energy model is a 2HDM. If $\alpha=0$, however, the linear term is zero automatically, and $\alpha_{3}$ can be of order 1, so that both charged Higgs boson masses in Eqs. (10) are naturally of order $v_{R}^{2}$. Put conversely, $\alpha_{3}$ $=\mathcal{O}(1)$ implies $\alpha=0$ and a heavy second Higgs multiplet, while SCPV requires a small $\alpha_{3}$ and thereby implies a second light doublet. Hence the $C P$ properties of the vacuum state are connected to the Higgs spectrum in the decoupling limit. We further stress that the scenario with $\alpha=0$ cannot be obtained by taking the limit $\alpha \rightarrow 0$ from the general case. For $\alpha=0$ and $\alpha_{3}=\mathcal{O}(1)$ we find a SM-like Higgs spectrum for $v_{R} \rightarrow \infty$ in agreement with $[6,9,10]$. However, we again face a fine-tuning problem, because in Eqs. (7) the three equations only involve two parameters $v_{R}$ and $k_{2} / k_{1}$. By eliminating $v_{R}$ and $k_{2} / k_{1}$ from Eqs. (7) one easily finds a relation between $\mu_{1,2,3}, \alpha_{1,2,3}$ and $\rho_{1}$ which cannot be justified by any symmetry.

\section{GENERAL SCENARIOS}

Next we will study general scenarios with $v_{L} \neq 0$. We solve the minimization conditions for $\mu_{1}^{2}, \mu_{2}^{2}, \rho_{1}, \rho_{3}, \beta_{1}$, and $\beta_{2}$. The solutions for $\mu_{1}, \mu_{2}$ and $\rho_{1}$ can be found in Eqs. (7). For $\rho_{3}, \beta_{1}$ and $\beta_{2}$ we find:

$$
\begin{aligned}
& \rho_{3}=\frac{\alpha_{3} \sin ^{2} \alpha k_{1}^{2} k_{2}^{2}}{v_{L}^{2}\left\{k_{1}^{2} \sin ^{2} \theta-k_{2}^{2}\left[\sin ^{2} \alpha+\sin ^{2}(\alpha+\theta)\right]\right\}}+\mathcal{O}\left(\epsilon^{0}\right), \\
& \beta_{1} \simeq \rho_{3} \frac{v_{L} v_{R}}{k_{1} k_{2}} \frac{\sin \theta}{\sin \alpha}, \quad \beta_{2} \simeq-\beta_{1} \frac{k_{2}}{k_{1}} \frac{\sin (\alpha+\theta)}{\sin \theta} .
\end{aligned}
$$

If both $\alpha$ and $f \equiv k_{1} k_{2} /\left(v_{L} v_{R}\right)$ are of order 1 , one faces non-perturbatively large couplings $\beta_{1}, \beta_{2}, \rho_{3}=\mathcal{O}\left(\epsilon^{-2}\right)$, unless $\alpha_{3}=\mathcal{O}\left(\epsilon^{2}\right)$. Equations (12) can be viewed as a seesaw relation between $1 / \alpha_{3}$ and $\beta_{1}, \rho_{3}$. The pivot is proportional to $\sin \alpha$, so that the effect vanishes in the absence of the $C P$-violating phase. Its origin can be traced back to the terms in $V$ which involve $\phi_{2}^{0 i}$, as in Eq. (11). Hence we confirm the findings of $[6,7]$ that $\alpha=\mathcal{O}(1)$ and $k_{1} \sim k_{2}$ enforces the smallness of parameters in the potential. However, this feature also appears in all other scenarios, as we discussed above. Hence an exhaustive investigation of the Higgs sector requires us to consider all these possibilities that $C P$ phases, ratios of VEVs and some of the Higgs couplings scale with certain powers of $\epsilon$. We have performed a complete analysis of all possible scenarios and discuss the generic cases here.

\section{Small $k_{2} \sin \alpha$}

If $\rho_{3}$ and $\alpha_{3}$ are of order 1, Eqs. (12) show that $\left(k_{2} / k_{+}\right) \sin \alpha$ is $\mathcal{O}\left(\epsilon^{2}\right)$ or smaller. For $\left(k_{2} / k_{+}\right) \sin \alpha$ $=\mathcal{O}\left(\epsilon^{2}\right)$ the triplet phase $\theta$ must be of order 1. Smaller values of $\theta$ are correlated with even tinier values of $\alpha$. Interestingly, one now finds the relation:

$$
2 \rho_{3}-\rho_{1}=\mathcal{O}\left(\epsilon^{2}\right)
$$

That is, in this scenario the electroweak scale is defined by $k_{+}^{2}=\mathcal{O}\left[\left(2 \rho_{3}-\rho_{1}\right) v_{R}^{2}\right]$, while $v_{R}^{2}$ is as usual defined by $\mu_{3}^{2} / \rho_{1}$ through Eqs. (7). In any grand unified theory (GUT) scenario in which $\Delta_{L}$ and $\Delta_{R}$ belong to the same GUT multiplet, $2 \rho_{3}-\rho_{1}$ vanishes exactly above the GUT scale. Below the GUT scale $2 \rho_{3}-\rho_{1}$ acquires a small non-zero value from radiative corrections. Such a situation occurs for example in SO(10) models [10]. The responsibility of the GUT symmetry for the gauge hierarchy $k_{+} \ll v_{R}$ is certainly very interesting. However, the small parameter $2 \rho_{3}-\rho_{1}$ also proliferates into the mass matrices; the leading terms for the singly charged masses are as in Eqs. (10). While the doublet with the FCNC Higgs boson now becomes heavy, we instead face an extra light Higgs triplet, whose components are dominantly $\Delta_{L}$ fields. The explicit calculation of the neutral mass matrix, however, shows that the triplet masses are of order $v_{L}$ or smaller. Since at least one of the triplet fields couples to the Z-boson, this scenario would have been discovered at the CERN $e^{+} e_{-}$collider LEP I. These findings also hold for the case that $k_{2} \sin \alpha$ is exactly zero with $\theta$ $\neq 0$.

If both $\left(k_{2} / k_{+}\right) \sin \alpha$ and $\alpha_{3}$ are $\mathcal{O}(\epsilon)$, we find three light neutral Higgs bosons: one is SM-like and two are mixtures of the $\delta_{L}^{0}$ and the flavor-violating $\phi_{+}^{0}$. This scenario interpolates between the previous and the following one.

\section{Large $k_{2} \sin \alpha$}

For $\left(k_{2} / k_{+}\right) \sin \alpha=\mathcal{O}(1)$ the minimization conditions in Eqs. (12) require $\alpha_{3}=\mathcal{O}\left(\epsilon^{2}\right)$, thus $\alpha_{3}$ defines the gauge hierarchy here. This scenario agrees qualitatively with the one discussed above for $v_{L}=0$. As stated previously the smallness of $\alpha_{3}$ leads to a 2HDM in the decoupling limit, with unacceptable flavor-violating neutral couplings in Eq. (5). We have also calculated the renormalization group flow of the flavor-changing neutral Higgs couplings in order to rule out a possible suppression mechanism of these couplings.

\section{No SCPV}

For $\alpha=\theta=0$ the minimization conditions with respect to $\Delta_{L}^{0 i}$ and $\phi_{2}^{0 i}$, which implied the smallness of $\alpha_{3}$ or $2 \rho_{3}$ $-\rho_{1}$, vanish and we are just left with 4 minimization conditions. Now we can arrange the nonstandard Higgs bosons to decouple for $v_{R} \rightarrow \infty$. Also this scenario agrees qualitatively with the zero phase case with $v_{L}=0$ : Eqs. (7) imply a severe fine-tuning problem. After eliminating $v_{R}$ and $k_{2} / k_{1}$ from Eqs. (7) the parameters $\mu_{1,2,3}, \alpha_{1,2,3}$ and $\rho_{1}$ combine to a relation requiring that parameters of order 1 cancel up to terms of order $\epsilon^{2}$. This phenomenon is at the heart of the 
gauge hierarchy problem. One usually addresses it by choosing the mass parameters $\mu_{1,2}$ of the fields which break the electroweak symmetry to be of order $k_{+}$. That is, the gauge hierarchy $k_{+} \ll v_{R}$ is put into $V$ by hand. In our case this solution would also require to choose $\alpha_{i}=\mathcal{O}\left(\epsilon^{2}\right), i=1,2,3$ [see Eqs. (7)] leading again to a 2HDM.

\section{$C P$ VIOLATION IN $V$ AT A HIGH SCALE}

The essential prerequisite for the connection between the $C P$ phases and the Higgs spectrum found above is the spontaneous breakdown of $C P$ at the electroweak scale. Within the MLRM a new situation occurs, if one allows for explicit $C P$ violation in $V$. In this case only the term involving $\alpha_{2}$ in Eq. (2) changes [see Eq. (A2) of [6]] and $\alpha_{2}$ may be complex. To order $\epsilon^{0}$ this case can be mapped on the discussed one by rescaling $\phi_{2}^{0} \rightarrow \phi_{2}^{0} e^{-i \varphi}$ with $\varphi=\arg \left(\alpha_{2} v_{R}^{2} / 2-\mu_{2}^{2}\right)$. Hence the previous findings on the Higgs spectrum in the decoupling limit remain valid with the replacement $\alpha \rightarrow \alpha$ $+\varphi$. Now the SM-like spectrum occurs for $\alpha=-\varphi$. Going beyond the MLRM, one can add extra singlet Higgs fields with large VEVs of order $v_{R}$ [8]. Then spontaneous $C P$ phases can appear between different VEVs of order $v_{R}$, i.e. $C P$ is broken well above the electroweak scale. This case can be mapped on the case with explicit $C P$ violation, with $\varphi$ now being a calculable function of the new $C P$ phases. Phenomenological studies of CKM $C P$ violation in these models requires a renormalization group analysis of the Yukawa sector to account for the large logarithm $\ln \left(v_{R} / k_{+}\right)$.

\section{CONCLUSIONS}

We have determined the general Higgs potential of the MLRM in the decoupling limit $v_{R} \rightarrow \infty$ and established a strict connection between the $C P$ properties of the vacuum state and the Higgs spectrum: if any of the $C P$ phases $\alpha$ $+\varphi$ or $\theta$ is nonzero, the low-energy model always differs from the SM. Either a $2 \mathrm{HDM}$ with flavor-changing Higgs bosons or a model with additional light triplet fields emerges, both of which are phenomenologically ruled out. The appearance of the triplet Higgs model in the decoupling limit for small but non-zero $\alpha$ has not been discussed in previous analyses. In particular papers which simultaneously assume a large $\alpha+\varphi$ and a SM-like Higgs spectrum are not correct. An important difference from previous analyses of the Higgs sector is that our results hold generally, even for fine-tuned parameters. We found that fine-tuning arguments do not discriminate between scenarios with and without spontaneous $C P$ phases. Fine-tuning is unavoidable for the Higgs potential to produce the gauge hierarchy $v_{R} \gg k_{+}$. To obtain the SM in the decoupling limit in the case $\alpha+\varphi=\theta$ $=0$, a fine-tuning condition between $\mu_{1,2,3}, \alpha_{1,2,3}$ and $\rho_{1}$ implied by Eqs. (7) must be satisfied. This condition requires parameters of order $v_{R}^{2}$ to cancel up to terms of order $k_{+}^{2}$ and cannot be justified by an approximate symmetry. The main conclusion of our paper is that the MLRM does not allow for spontaneous $C P$ violation at the electroweak scale. Adding extra singlet fields does not change this conclusion, but opens the possibility for spontaneous $C P$ violation at the high scale $v_{R}$ parametrized by a new phase $\varphi$. Decoupling of the non-standard Higgs bosons then requires $\alpha=-\varphi$.

\section{ACKNOWLEDGMENTS}

This work is supported by BMBF under contract No. 05HT1WOA3, by the EU under the TMR contract No. HPMF-CT-2000-00460, and by ESF grants No. 3832 and 5135. Fermilab is operated by URA under DOE contract No. DE-AC02-76CH03000.
[1] J.C. Pati and A. Salam, Phys. Rev. D 10, 275 (1974); R.N. Mohapatra and J.C. Pati, ibid. 11, 566 (1975); 11, 2558 (1975); G. Senjanović and R.N. Mohapatra, ibid. 12, 1502 (1975); G. Senjanovic, Nucl. Phys. B153, 334 (1979); R.N. Mohapatra and G. Senjanović, Phys. Rev. D 23, 165 (1981); C.S. Lim and T. Inami, Prog. Theor. Phys. 67, 1569 (1982).

[2] M. Gell-Mann, P. Ramond, and R. Slansky, in Supergravity, edited by $\mathrm{P}$. van Nieuwenhuizen and D. Freedman (NorthHolland, Amsterdam, 1979); print-80-0576 (CERN); T. Yanagida, in Proceedings of the Workshop on the Unified Theory and the Baryon Number in the Universe, edited by $\mathrm{O}$. Sawada and A. Sugamoto, Tsukuba, Japan, 1979 (KEK Report No. 79-18, Tsukuba, 1979); R.N. Mohapatra and G. Senjanović, Phys. Rev. Lett. 44, 912 (1980).

[3] G. Barenboim, J. Bernabeu, and M. Raidal, Nucl. Phys. B511, 577 (1998); G. Barenboim, J. Bernabeu, J. Matias, and M. Raidal, Phys. Rev. D 60, 016003 (1999); P. Ball and R. Fleis- cher, Phys. Lett. B 475, 111 (2000).

[4] P. Ball, J.M. Frère, and J. Matias, Nucl. Phys. B572, 3 (2000).

[5] D. Chang, Nucl. Phys. B214, 435 (1983); G. Ecker, W. Grimus, and H. Neufeld, ibid. B247, 70 (1984); G. Ecker and W. Grimus, ibid. B258, 328 (1985); J.-M. Frère et al., Phys. Rev. D 46, 337 (1992); G. Barenboim et al., Nucl. Phys. B478, 527 (1996); G. Barenboim and J. Bernabeu, Z. Phys. C 73, 321 (1997).

[6] N.G. Deshpande, J.F. Gunion, B. Kayser, and F. Olness, Phys. Rev. D 44, 837 (1991).

[7] J. Basecq, J. Liu, J. Milutinovic, and L. Wolfenstein, Nucl. Phys. B272, 145 (1986).

[8] G.C. Branco and L. Lavoura, Phys. Lett. 165B, 327 (1985).

[9] P. Duka, J. Gluza, and M. Zralek, Ann. Phys. (N.Y.) 280, 336 (2000).

[10] A. Datta and A. Raychaudhuri, Phys. Rev. D 62, 055002 (2000). 